\title{
Socrative como herramienta para la evaluación y aprendizaje de Fundamentos Matemáticos en el estudiantado universitario
}

Socrative as a tool for the evaluation and learning of Mathematical

Foundations in university students

\author{
Volumen 22 Número 1 \\ Enero - Abril \\ pp. 1-29
}

\begin{abstract}
Niorka Medina Cepeda José Delgado Fernández Reinaldo Guerrero Chirinos
\end{abstract}




\title{
Socrative como herramienta para la evaluación y aprendizaje de Fundamentos Matemáticos en el estudiantado universitario
}

Socrative as a tool for the evaluation and learning of Mathematical Foundations in university students

\author{
Niorka Medina Cepeda ${ }^{1}$ \\ José Delgado Fernández ${ }^{2}$ \\ Reinaldo Guerrero Chirinos ${ }^{3}$
}

\begin{abstract}
Resumen: El bajo rendimiento académico en el área de matemáticas a nivel universitario, es preocupante en países de Latinoamérica como Ecuador y Venezuela. Por este motivo, cobra mayor fuerza la presencia de herramientas educativas innovadoras, las cuales, mediante dispositivos móviles, facilitan la evaluación permitiendo monitorear el progreso del estudiantado en su aprendizaje. El objetivo general de este artículo fue, analizar el uso de Socrative como herramienta para la evaluación y el aprendizaje de Fundamentos Matemáticos en el estudiantado universitario. Para ello, se utilizó una metodología cuantitativa, de tipo descriptivo, con un diseño de investigación de campo, transversal, cuya población fue de 542 estudiantes inscritos, y la muestra fue de 36 estudiantes pertenecientes al primer ciclo académico del periodo Octubre 2018-Febrero 2019 de la Universidad Técnica Particular de Loja, en la ciudad de Loja en Ecuador; los instrumentos fueron cuestionarios elaborados con Socrative y una encuesta de satisfacción aplicada al estudiantado. Los resultados indicaron que la herramienta Socrative resultó ser sobresaliente y beneficiosa para la evaluación y el aprendizaje de la unidad Funciones, donde el estudiantado mostró un progreso en sus niveles de conocimiento y en sus calificaciones. Además, el alumnado reconoció que este recurso propicia el desarrollo de sus habilidades y el trabajo autónomo. Se concluyó que Socrative representa una herramienta didáctica de gran potencial para la mejora del rendimiento estudiantil, pues, mediante la evaluación en diferentes momentos, se logró percibir las debilidades y fortalezas que se presentan en el proceso de aprendizaje, con la intención de enmendar tales debilidades en el momento propicio.
\end{abstract}

Palabras clave: método de evaluación - Socrative, evaluación, aprendizaje, matemáticas

\begin{abstract}
The low academic performance in the area of mathematics at the college level is worrying in Latin American countries such as Ecuador and Venezuela. This is why the presence of innovative educational tools, which through mobile devices, facilitate evaluation, allowing monitoring of the student's progress in their learning. The general objective of this article was to analyze the use of Socrative as a tool for the evaluation and learning of Mathematical Foundations in university students. For this, a quantitative, descriptive methodology was used, with a cross-sectional field research design, whose population was 542 enrolled students and the sample was 36 students belonging to the first academic cycle of the October 2018-February 2019 period; the instruments were questionnaires elaborated with Socrative and a satisfaction survey applied to the students. The results indicated that the Socrative tool turned out to be outstanding and beneficial for the evaluation and learning of the Unit Functions, the students showed progress in their levels of knowledge and in their grades. In addition, they recognized that this resource encourages the development of their skills and autonomous work. It was concluded that Socrative represents a didactic tool with great potential for the improvement of student performance, because through the evaluation at different moments it was possible to perceive the weaknesses and strengths that are presented in the learning process with the intention of amending at the right moment.
\end{abstract}

Key Words: evaluation methods - Socrative, evaluation, mathematics, learning

1 Universidad del Zulia, Maracaibo, Venezuela. Dirección Electrónica: niorkamedina@gmail.com ORCID: https://orcid.org/0000-0001-7023-0635

2 Universidad Técnica Particular de Loja, Loja, Ecuador. Dirección Electrónica: jrdelgado66@utpl.edu.ec ORCID: https://orcid.org/0000-0002-9176-7666

3 Universidad del Zulia, Maracaibo, Venezuela. Dirección Electrónica: reinaldoguerrero1970@gmail.com ORCID: https://orcid.org/0000-0003-0499-7453

Artículo recibido: 29 de junio, 2021

Enviado a corrección: 18 de octubre, 2021

Aprobado: 6 de diciembre, 2021

Los contenidos de este artículo están bajo una licencia Creative Commons 


\section{Introducción}

Actualmente se han presentado cambios relevantes en la manera de impartir las clases en las aulas universitarias debido a la presencia de nuevas estrategias didácticas apoyadas en el uso de las tecnologías de información y comunicación (TIC) las cuales facilitan el proceso evaluativo y; la supervisión del progreso y logros del alumnado de forma inmediata, lo que lleva a incentivar el trabajo colaborativo e, incluso, fomentar la motivación en el estudiantado (Basso y Loyer, 2016; Parra et al., 2017; Puche, 2018).

En este sentido, los dispositivos móviles son cada vez más comunes en el aula, pues representan nuevos recursos de apoyo al aprendizaje, lo que ha permitido que surja una nueva estrategia conocida como Mobile learning, la cual ha logrado un fuerte auge en las aulas (Frías et al., 2016; Masero, 2019); además, la mayoría del alumnado presenta habilidades en el manejo de herramientas digitales ya que es parte de su rutina pues nacieron en la denominada era digital.

Asimismo, el profesorado dispone de diferentes herramientas de libre acceso en internet que pueden ser descargadas en los dispositivos móviles, con las cuales pueden diseñar sus propios exámenes de manera electrónica y aplicarlos en línea; este tipo de plataformas forman parte de los llamados sistema de respuesta estudiantil o sistema de respuesta a audiencia, SRS (Fies y Marshall, 2006). Dichos sistemas resultan de gran ayuda, pues, mediante la retroalimentación instantánea que ofrecen sobre el progreso del alumnado, se pueden detectar y corregir los errores en el tema tratado, tomar decisiones sobre la dirección del aprendizaje, e, incluso, el profesorado puede reforzar, modificar o modernizar sus estrategias metodológicas en clases en caso de que lo ameriten (López et al., 2017; Masero, 2019).

Ante estas circunstancias, el reto es aprovechar las bondades que estas herramientas digitales ofrecen en el escenario universitario. Por tal motivo, en esta investigación se ha analizado a la plataforma Socrative como herramienta para la evaluación y aprendizaje del estudiantado universitario, una aplicación de acceso libre que permite la creación de diversos tipos de cuestionarios online, los cuales suelen representar nuevas actividades didácticas y evaluaciones que pueden ser aplicadas en diferentes momentos del proceso de aprendizaje mediante dispositivos móviles en tiempo real.

De este modo, al realizar las evaluaciones en distintos momentos de la clase y recibir sus resultados de manera inmediata, se podrá diagnosticar los conocimientos previos del estudiantado, monitorear el avance del aprendizaje durante el desarrollo de los contenidos y, finalmente, verificar si al cierre de la clase se lograron los objetivos de aprendizaje trazados 
(Grueso et al., 2016; Pianucci y Tenutto, 2020). En consecuencia, tanto el profesorado como el estudiantado podrán reflexionar y tomar acciones para mejorar el proceso de enseñanzaaprendizaje en los diferentes momentos de la clase. La evaluación se convertirá, entonces, en un componente central en la efectividad del proceso de aprendizaje promoviendo prácticas de aprendizaje activas en un ambiente divertido, cómodo y social en el que el estudiantado universitario se sienta comprometido con su proceso de aprendizaje (Jaber et al., 2018; Santos et al., 2016).

Entre los autores que han realizado investigaciones relacionadas con la utilización de la plataforma Socrative como herramienta de apoyo al proceso de aprendizaje se encuentran Frías et al. (2016), Parra et al. (2017) y Narbón y Peiró (2018), cuyas conclusiones indicaron un aumento en la participación activa del estudiantado, lo que permitió mejorar considerablemente su aprendizaje y su ambiente de clase, su motivación se incrementó así como la atención en las clases teóricas. Según los estudios elaborados por Rapún y Rapún (2018), Moya y Soler (2018), y Cosi y Voltas (2019), el alumnado expresó que con el uso de esta plataforma se logra una mayor asimilación de los tópicos teóricos-prácticos lo que le permite realizar un el autoanálisis sobre sus habilidades, capacidades y conocimientos; además el estudiantado también señaló que al utilizar esta herramienta se fortalece la relación con su docente y entre el estudiantado.

Por otro lado, Perera y Hervás (2019) y Subirats (2018) consideraron que con esta aplicación se logra establecer una conexión entre el ambiente digital en el que se desenvuelve el alumnado y el contexto formal de la universidad en la que estudia, lo cual resulta una experiencia de aprendizaje más personalizada. Para los investigadores Rodríguez et al. (2017) y Chamorro et al. (2021), el gran aporte del uso de esta aplicación viene dado por el incremento del grado de atención del estudiantado en clase, la motivación y el interés hacia la asignatura; así como su efectividad para detectar confusiones particulares en el tema tratado o fallas generales.

Todo lo anteriormente expuesto supone un gran incentivo para introducir estrategias didácticas de innovación metodológicas y evaluativas en el campo de la matemática universitaria. Particularmente, se ha obtenido un bajo rendimiento académico en la unidad Funciones de la asignatura Fundamentos Matemáticos que se imparte en la Universidad Técnica Particular de Loja, ubicada en la ciudad de Loja en el Ecuador. En consecuencia, resulta importante indagar si entre las causas de este bajo rendimiento se encuentra que el profesorado no utiliza las herramientas metodológicas más adecuadas para impartir esta 
asignatura. Basado en ello, surge la siguiente interrogante: ¿cómo sería el aporte de la plataforma Socrative en el proceso de evaluación y de aprendizaje de la unidad Funciones de la asignatura Fundamentos Matemáticos en estudiantado universitario?

Ante tal situación y con la intención de crear un ambiente en clase en el que el estudiantado sea protagonista de su aprendizaje y pueda desarrollar su pensamiento lógicoreflexivo; se presenta un estudio cuyo objetivo general consiste en analizar el uso de Socrative como herramienta para la evaluación y el aprendizaje de Fundamentos Matemáticos en el estudiantado universitario. Para ello los objetivos específicos de esta investigación se enfocan en determinar: el nivel de conocimientos previos en estudiantes, el nivel de avance en el aprendizaje del estudiantado, el nivel de comprensión del estudiantado y, finalmente, el grado de satisfacción del estudiantado sobre el uso del Socrative en situaciones de evaluación y de aprendizaje.

La estructura del presente artículo viene dada por: tras la introducción, una segunda sección sobre los referentes teóricos de la investigación en la que se desarrollan los fundamentos teóricos que soportan la presente investigación; la tercera sección la compone la metodología que incluye la descripción de: el enfoque, las unidades de análisis, el contexto, las técnicas de recolección y el procesamiento de análisis de los datos; la cuarta sección los resultados y su discusión según autores; y finalmente, el apartado de las conclusiones.

\section{Referentes Teóricos}

\subsection{El proceso de evaluación de aprendizajes}

Al hablar de evaluación en el campo educativo, en cualquiera de sus niveles, no se puede dejar de asociarla a dos de sus funciones: la formativa y la certificadora; convirtiéndola en uno de los tópicos con mayores controversias y; que ha sido y será el centro de muchas investigaciones. De hecho, buena parte de estos estudios han verificado que la evaluación representa uno de los elementos esenciales dentro del proceso de enseñanza aprendizaje (Arribas, 2017; Díaz et. al., 2018).

En consecuencia, surgen varias inquietudes sobre cómo convertir a la evaluación en una herramienta para optimizar el aprendizaje sin que se pierdan sus características de validez y confiabilidad al verificar que se han logrado los objetivos y competencias trazadas en el proceso de enseñanza aprendizaje. Razón por la cual, se considera que "la evaluación alcanza su sentido máximo cuando es utilizada como un medio de aprendizaje y no como un fin" (Díaz et al., 2018, p.179). Sin duda alguna, para estos mismos autores, la evaluación como 
instrumento pedagógico es la mejor vía para medir los conocimientos siempre y cuando no se realice de manera inadecuada pues generaría apatía en el estudiantado y por ende, rechazo.

En este sentido, la clave de interpretar a la evaluación como un recurso beneficioso en el proceso de enseñanza, es facilitar la creación de un camino de comunicación basado en el diagnóstico, la corrección y modificación de aquellas estrategias y actividades que así lo ameriten, permitiendo la retroalimentación al informar al estudiantado sobre su progreso para conducirlos a aprendizajes significativos (Jordán et al., 2018).

De esta manera, cuando docentes y estudiantes están al tanto de sus debilidades y fortalezas en la práctica educativa se genera suficiente insumo para reflexionar y realizar la intervención pedagógica adecuada dentro del mismo proceso evaluativo, aprendiendo así de sus propios errores y tomándolo como principio para la transformación de paradigmas. Sin duda alguna, que el propósito en común será confirmar o rectificar las estrategias utilizadas y guiar al estudiantado sobre la misma marcha para que avance y mejore su aprendizaje (Sánchez, 2018).

Por consiguiente, si se toma en consideración que el "aprendizaje y evaluación son inseparables y que ésta favorece un proceso de aprendizaje constructivo, reflexivo y eficaz..." (Fernández, 2017, p.12), es necesario seleccionar recursos metodológicos innovadores de evaluación acorde con ese propósito, con los cuales se motive al estudiantado para captar su atención y participación. Es por ello que para Arraiga et al. (2017), la integración de herramientas tecnológicas en el aula representa un gran apoyo y aporte en la fortificación de una conveniente evaluación y un alto nivel de aprendizaje.

\subsection{Mobile Learning}

Actualmente, es común el uso de las tecnologías dentro de las aulas universitarias. Por tal motivo, el profesorado universitario tiene como reto formarse continuamente para la renovación de sus estrategias metodológicas con el objeto de ofrecerle al estudiantado un ambiente caracterizado por la innovación y eficacia en el que se garantice un aprendizaje competitivo y activo, siendo el estudiantado responsable y protagonista de su propio aprendizaje; además de brindarle una clase impregnada de motivación que favorezca el aprendizaje significativo (Puche, 2018).

No obstante, se debe considerar que la utilización de las TIC debe responder a un objetivo específico en el que se busque la solución a una situación problema o mejora del proceso de enseñanza aprendizaje fuera o dentro del salón de clases; pues su utilización no 
debe representar una simple "decoración" o "moda" dentro del contexto educativo (Rodríguez et al., 2017). De allí, la importancia de la selección adecuada de la herramienta digital a utilizar, ya que debe cubrir y adaptarse a una determinada situación de aprendizaje (Moya y Soler, 2018).

Bajo estas circunstancias, hoy en día, existen una serie de herramientas tecnológicas educativas que logran fortalecer el proceso de enseñanza, aprendizaje y evaluación y, son conocidas como mobile learning. Estas nuevas alternativas pedagógicas están relacionadas con la integración de los dispositivos móviles (teléfonos inteligentes, tabletas, ordenadores portátiles) en el aula, en pro de una comunicación más fluida y continua como apoyo para la construcción de un conocimiento de una forma sencilla, rápida y divertida; y así obtener un aprendizaje activo, abierto y compartido que pueda ser evaluado en tiempo real (López y Silva, 2016; Zamora, 2019).

Hay que considerar que el actual estudiantado universitario, representa a los llamados nativos digitales, con una gran destreza en el uso de dispositivos móviles y del internet como medio hacia el conocimiento. Aunado a ello, la posibilidad de acceso a los contenidos en cualquier momento y lugar favorece considerablemente un aprendizaje flexible y personalizado permitiendo que estas nuevas experiencias educativas adquieran mayor importancia (Mateus et al., 2017).

La mayoría de estos alumnos dispone de su propio dispositivo móvil, lo cual representa un beneficio para el profesorado, quienes pueden planificar actividades en línea en distintos momentos de su clase (Zamora, 2019). De esta manera, cada docente deberá mantener su papel como orientador en el proceso de aprendizaje así como en la búsqueda, creación y diseño de material didáctico digital pertinente, según los objetivos de aprendizaje, impulsándolo a capacitarse y actualizarse en el manejo de herramientas informáticas de uso educativo.

Otra de las bondades de utilizar los móviles en clase es que se caracterizan por ser herramientas de respuesta de audiencia en la que el estudiantado no requiere de aparatos distintos a los que cotidianamente utiliza (López et al., 2019).

Por otro lado, existen aplicaciones para dispositivos móviles que pueden desarrollar con mayor efectividad estrategias para captar la atención y alerta del estudiantado en diferentes momentos de la clase bien sea para repasar, reflexionar o evaluar los conocimientos adquiridos; siendo de acceso gratuito y sencillo, facilitándole al profesorado la tarea de implementar un aula virtual o bien haciendo más dinámica a una clase convencional, en la que 
el estudiantado pueda contestar preguntas y se muestren los resultados de manera inmediata (Rodríguez et al., 2017). En este sentido, el éxito de estas aplicaciones consiste en cómo lograr su mejor contribución en el aprendizaje más que en la forma de integrarlas en la enseñanza universitaria (López y Silva, 2016).

En consecuencia, con la utilización de los dispositivos móviles en el aula están surgiendo nuevos escenarios de formación, los cuales le brindan a al estudiantado un ambiente de aprendizaje con mayor motivación, participación en las que se pueda obtener una autorregulación de su propio aprendizaje. De esta manera, se logra que el alumnado acepte de forma positiva la integración de estos recursos en la clase ya que pueden combinar actividades como la autoevaluación mediante test o cuestionarios; y el aprendizaje a través de juegos competitivos (Narbón y Peiro, 2018; Perera y Hervás, 2019).

\subsection{Sistemas de Respuesta del Estudiantado (SRS)}

Los sistemas de respuesta del estudiantado (SRS) también conocido como sistema de respuesta a audiencia, permiten que el profesorado formule preguntas para ser respondidas por el estudiantado obteniendo de forma inmediata una retroalimentación sobre los resultados; es decir, existe un feedback en tiempo real. Igualmente, estos sistemas generan información cualitativa y estadística mediante un reporte al que tendrán acceso directo el profesorado y en el que se visualizará el avance en el aprendizaje de cada estudiante (López et al., 2017; Moya y Soler, 2018). Asimismo, debido a que el estudiantado tiene mayor accesibilidad a la web, éstos pueden interactuar con una plataforma, de acceso libre, en el que se le propongan cuestionarios o test relacionados con contenidos específicos de una manera rápida y entretenida. A estas herramientas virtuales pertenece Socrative.

Entre las ventajas que ofrecen estos sistemas en el campo educativo se encuentran: facilita el aprendizaje activo, incrementa la interacción en el aula, fomenta la motivación del alumnado y su autoevaluación, aumenta la participación activa, colaborativa y competitividad de los alumnos en clase, contribuye a un mayor protagonismo del estudiantado en su aprendizaje, promueve el aprendizaje colaborativo, se recibe y se comprende la retroalimentación de resultados en la misma clase, el profesorado conoce el avance y comprensión de los contenidos por parte del estudiantado de forma rápida, la evaluación y autoevaluación es de manera continua, permite identificar los tópicos que deben repasarse con mayor detalle, representa una herramienta de evaluación diagnóstica (Masero, 2019; Parra et al., 2017). 
Entre sus desventajas se distingue que con el paso del tiempo y debido al dominio de la asignatura, al uso continuo de los dispositivos y el número de asistencia a clase; el estudiantado suele aburrirse y su opinión positiva sobre el uso de estas herramientas suele cambiar, por lo tanto se sugiere alternar y variar el uso de diferentes herramientas virtuales de acuerdo al objetivo de aprendizaje a lograr (Moya y Soler, 2018).

\subsection{Socrative como herramienta para la evaluación y el aprendizaje.}

La plataforma Socrative constituye una herramienta online de acceso gratuito, en la que el profesorado crea su propia cuenta para diseñar cuestionarios bajo las modalidades: verdadero/falso, respuesta múltiple y respuesta corta. El profesorado y el alumnado pueden conectarse a través de móviles, bien sea celular, tableta o laptop, en el momento de la clase, es decir en tiempo real. Esta plataforma fue creada en el año 2011 por Berté, West y Duncan (Frías et al., 2016).

El procedimiento es muy sencillo, ya que el profesorado está en la posibilidad de asignar una o varias preguntas durante la clase relacionadas con algún contenido explicado durante la misma clase o con contenidos explicados en clases anteriores con el propósito de introducir, reforzar o profundizar en un tema en específico. Su descarga es rápida y sencilla, el profesorado le indica al alumnado una clave para que pueda acceder a la sala y tener a su disposición los cuestionarios a realizar.

De esta manera, la participación del estudiantado en la clase se incrementa, se evalúa constantemente por tanto se incentiva a la evaluación sumativa y el profesorado puede observar el avance individual o en grupo en tiempo real. Al finalizar la actividad, la cual se cierra al vencerse el tiempo programado por el profesorado, la plataforma le ofrece la posibilidad de acceder a la estadística sobre el resultado del trabajo realizado en el aula, mostrándole al profesorado el porcentaje y número de respuestas correctas en general y por pregunta; así como el promedio de respuestas acertadas por el curso. Por tal motivo, el grupo profesoral puede tomar los correctivos que se requieran para mejorar el proceso de aprendizaje.

Las actividades de evaluación, mediante esta herramienta, pueden llevarse a cabo en diferentes momentos de la clase, dependiendo el objetivo que se pretende alcanzar. De hecho, una de sus bondades es que la base de datos de las preguntas del cuestionario, por tema a evaluar pueden responderse hasta en menos de 10 minutos de forma dinámica y entretenida (Parra et al., 2017). Esta herramienta le ofrece al alumnado una experiencia de aprendizaje 
basada en un conjunto de retos cognitivos presentada de forma amena dado la variedad de materiales digitales que se les puede integrar; y el alumnado logra tener un control de su aprendizaje promoviendo el debate y la participación activa (Perera y Hervás, 2019).

Otro aporte es que el estudiantado puede diseñar sus propios cuestionarios con tópicos correspondientes al tema a evaluar, pues la plataforma ofrece la posibilidad que los test pueden ser diseñados por docentes o bien por estudiantes (Calderón et al., 2019).

Uno de los elementos relevantes es que se puede integrar los dispositivos móviles al aula, como por ejemplo los teléfonos móviles. Otra de sus características es que para formular las preguntas se cuenta con un número ilimitado de caracteres, por lo cual es más flexible que otras herramientas parecidas (Narbón y Peiró, 2018). Además, es de fácil descarga y uso, ya que el usuario no amerita de conocimientos explícitos sobre informática y aspectos técnicos; y su acceso es gratuito sin ningún requisito extraordinario (Moya y Soler, 2018; Puche y Pérez, 2018).

Por otro lado, ofrece una retroalimentación en tiempo real, con una interacción total, una visualización inmediata de los resultados los cuales pueden ser o no mostrados en clase según decisión del profesorado, quien puede ocultar o no los nombres del estudiantado. Asimismo, el profesorado puede recopilar información y gestionarla de la manera que mejor convenga y en el momento que lo requiera, permitiendo perfeccionar el proceso de enseñanza aprendizaje (Basso y Loyer, 2016; Calderón et al., 2019).

Entre sus bondades también se encuentra que fomenta el trabajo colaborativo, pues facilita la competición sana entre equipos y una participación activa del grupo en su proceso de aprendizaje, ya que genera un intercambio de puntos de vistas sobre la posible respuesta correcta una vez planteados los cuestionarios (López et al., 2019).

En resumen, la implementación del Socrative facilita la integración del proceso evaluativo en la actividad educativa diaria, haciendo énfasis en la reflexión, lo que permite que el estudiantado y el cuerpo profesoral tracen una ruta de aprendizaje, de modo de poder realizar los ajustes que se requieren. Por tal motivo, esta aplicación se convierte en una valiosa herramienta de evaluación, tanto para el diagnóstico de los conocimientos previos, el nivel de avance o progreso del alumnado durante la clase y finalmente, el nivel de comprensión general del contenido explicado. Durante todo este proceso evaluativo es posible contemplar la evolución del aprendizaje del estudiantado y hace factible realizar los cambios convenientes para incentivar ese aprendizaje (Chamorro et al., 2021). 


\section{Metodología}

\subsection{Enfoque}

La investigación presentó un enfoque cuantitativo para llevar a cabo el análisis de los datos. Con respecto al diseño de investigación, fue de campo, debido a que la información se recolectó directamente en las aulas de clases de la Universidad Técnica Particular de Loja (UTPL) ubicada en la ciudad de Loja en el Ecuador; además fue de tipo transversal, ya que los datos fueron recabados en un solo momento en el primer ciclo académico del periodo Octubre 2018-Febrero 2019. Según su alcance, fue de tipo descriptiva pues se identificó, describió y analizó el uso de la herramienta Socrative para la evaluación y aprendizaje de la unidad funciones en el estudiantado universitario de la asignatura Fundamentos Matemáticos.

\subsection{Unidades de análisis}

La población estuvo compuesta por 542 estudiantes inscritos en la cátedra Fundamentos Matemáticos pertenecientes a los 14 paralelos del primer ciclo académico del periodo Octubre 2018-Febrero 2019; de los cuales se tomó una muestra de 36 estudiantes inscritos en dicha cátedra, 25 del género masculino $(69,4 \%)$ y 11 del género femenino $(30,6 \%)$, en edades comprendidas entre 17 y 22 años, distribuidos en las carreras de Bioquímica y Farmacia, y de Ingeniería Química, correspondientes al área Biológica y Biomédica de la Universidad Técnica Particular de Loja. Esta muestra se seleccionó de manera no probabilística, por oportunidad. Los participantes que llenaron la encuesta manifestaron su compromiso de confidencialidad.

\subsection{Técnica de recolección}

Con el propósito de darle respuesta a las inquietudes planteadas durante la investigación se consideraron los siguientes instrumentos:

\subsubsection{Aplicación Socrative}

Para el inicio de tres clases, se diseñaron cuestionarios (test) constituidos por 10 preguntas: 5 de tipo opción múltiple y 5 de tipo verdadero/falso. En el momento del desarrollo de dos clases se lanzó una pregunta de respuesta corta y finalmente, al concluir los contenidos de la unidad. En el momento del cierre de la última clase, se utilizó la opción de carrera espacial (space race) compuesta por 10 ítems: 4 de verdadero/falso, 6 de selección simple (test). Estos cuestionarios fueron diseñados por los autores de este artículo, su validación estuvo en manos de docentes pertenecientes al departamento Química y Ciencias Exactas de la UTPL y del 
departamento de Matemáticas de la Facultad de Ingeniería de la Universidad del Zulia de Venezuela.

\subsubsection{Encuesta de Satisfacción sobre el uso del Socrative dirigido a Estudiantes} (ESSE)

Este cuestionario se diseñó con el propósito de conocer el nivel de satisfacción del estudiantado sobre la utilización de la plataforma Socrative. Para medir la confiabilidad de este instrumento se aplicó alfa Cronbach, cuyo resultado indicó una alta confiabilidad $(0,989)$. Tal cuestionario fue elaborado mediante la aplicación formulario de google; con 20 ítems de escala de tipo Likert, se midió la opinión del alumnado sobre el uso de esta plataforma mediante los siguientes criterios: Reforzar conocimiento, Motivación, Creatividad, Autoaprendizaje, Liderazgo, Trabajo cooperativo, Diversión, Razonamiento Crítico, Iniciativa Propia, Competencia Amigable, Desarrollo de Habilidades, Compromiso con la asignatura, Trabajo autónomo, Autocorrección, Visualización del progreso, Resolución de ejercicios y/o problemas, Feedback en tiempo real, Evaluación Continua, Evaluación de conocimientos previos, Rendimiento Final. Se utilizaron las siguientes alternativas de respuestas para la escala Likert: 1: Pésimo, 2: Malo, 3: Regular, 4: Bueno, 5: Excelente. Además, se incluyó una pregunta abierta que les permitía al estudiantado realizar observaciones alusivas a la incorporación de esta plataforma en próximas asignaturas.

\subsection{Procesamiento de Análisis}

El proceso estadístico de los datos obtenidos en la recolección se realizó mediante el programa SPSS versión 25. A continuación se detallan los procedimientos para medir a cada una de las dimensiones que componen el estudio: nivel de conocimiento previo en estudiantes, nivel de avance en el aprendizaje, nivel de comprensión en general y grado de satisfacción en estudiantes.

\subsubsection{En cuanto al nivel de Conocimiento}

Para este estudio se consideraron tres tipos de niveles de conocimiento del estudiantado, los cuales dependían del momento en que se encontraba la clase (inicio, desarrollo y cierre). Estos tipos de niveles fueron: previo, de avance y de comprensión en general. 
Para medir el nivel de conocimientos previos, se suele utilizar cuestionarios o test de diagnósticos o bien plantear preguntas en los primeros momentos de la clase que facilitarán el repaso de los contenidos de la sesión de aprendizaje anterior y contextualizar los conceptos así como preparar al estudiantado para las explicaciones que vienen a continuación e identificar las nociones esenciales para cada tema (Sánchez, 2018).

La medición del nivel de avance o progreso en el aprendizaje se puede realizar mediante actividades evaluativas de monitoreo como el planteamiento de preguntas sobre contenidos durante el desarrollo de una clase, por medio de preguntas directas a la clase o cuestionarios interactivos con retroalimentación inmediata de los resultados. De esta manera, el profesorado puede tomar decisiones sobre el control de las actividades pedagógicas como avanzar o no con el desarrollo de la explicación, el tiempo de dedicación, tipo de estrategias metodológicas, tipo de evaluación que realizará, entre otras (Joya, 2020; Saltos-Dueñas y ChiribogaZambrano, 2016).

En lo referente al nivel de comprensión en general, su medición está relacionada con evaluaciones de carácter sumativa, en la que se utilicen herramientas evaluativas en la fase final de la unidad que contenga preguntas sobre los contenidos tratados durante la unidad o asignatura. Con este tipo de valoraciones se puede detectar déficits en el aprendizaje del temario explicado y evaluar el progreso del estudiantado alineado con los objetivos de aprendizaje de la asignatura (Rodríguez y Gracia, 2016). Para esta clase de evaluaciones se pueden utilizar instrumentos como cuestionarios dinámicos y de competición que permitan demostrar la teoría que el alumnado ya debe haber asimilado.

En esta investigación, para determinar los niveles de conocimientos que conforman los tres primeros objetivos específicos se consideraron los resultados de cada uno de los tres cuestionarios aplicados al estudiantado con el uso del Socrative (inicial, intermedio y final), en diferentes momentos de la clase. Esos resultados fueron analizados bajo dos procedimientos estadísticos. En primer lugar, basándose en el número de aciertos de las preguntas en los cuestionarios (con un máximo de 10 aciertos, para cada uno), cada estudiante fue ubicado en uno de los 5 niveles de aprendizaje, de acuerdo con sus modelos de pensamiento (Santos et al., 2016). En la Figura 1, se muestra una escalera que representa los 5 niveles (A, B, C, D y E), los cuales varían según el grado de conocimiento demostrado por cada estudiante, siendo A el nivel más alto y $E$ el nivel de aprendizaje más bajo. 


\section{Figura 1}

Representación de los niveles de aprendizaje según modelos de pensamiento del estudiantado correspondientes a los cuestionarios aplicados

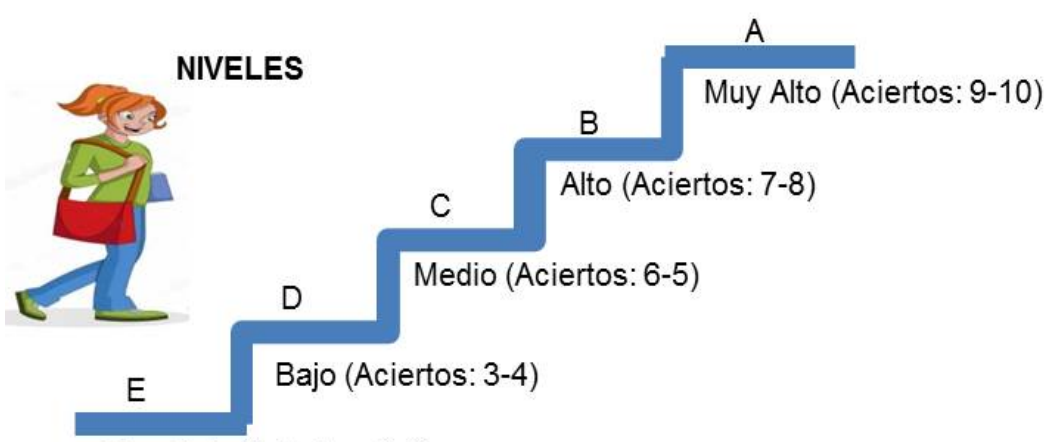

Muy Bajo (Aciertos: 0-2)

Fuente: Elaboración Propia, con información de Santos et al., 2016

En segundo lugar, para medir de manera general el progreso del alumnado en relación con el grado de comprensión de los contenidos de la unidad Funciones entre cada uno de los tres cuestionarios, se aplicó la Prueba de McNemar, la cual permitió constatar el progreso o no del nivel de conocimiento del estudiantado. Esta prueba es utilizada para decidir si se acepta o no que un determinado tratamiento (para este estudio, el uso del Socrative) induzca un cambio en la respuesta dicotómica (en este caso, satisfactorio o insatisfactorio) de los factores que están siendo expuestos a tal tratamiento (para la investigación, el nivel de conocimiento del estudiantado).

De esta manera, esta prueba permitió comprobar si con el uso del Socrative cada tipo de nivel de conocimiento del alumnado inducía un cambio en el siguiente nivel de conocimiento (de previo a avance, de avance a comprensión general) a medida que el alumnado estudiaba la unidad. Para realizar este análisis, se formuló la hipótesis nula: el resultado de un tipo de nivel de conocimiento (previo o de avance) del estudiantado no induce cambios significativos en la respuesta del siguiente tipo de nivel de conocimiento (de avance o de comprensión general) del estudiantado que utiliza Socrative, tal hipótesis se acepta si $p>.05$. Mientras que la hipótesis alternativa era que el resultado de cierto tipo de nivel de conocimiento (previo o de avance) del estudiantado si induce cambios en las respuestas del siguiente tipo de nivel de conocimiento (de avance o de comprensión general) del estudiantado que utiliza Socrative, la cual se acepta si $p<.05$. 


\subsubsection{En cuanto a la metodología empleada}

Para evaluar si la metodología utilizada (uso del Socrative), intervino en el resultado de las calificaciones o si éstas fueron al azar, se tomaron en cuenta las calificaciones del examen final de funciones de dos grupos: el grupo que utilizó socrative y otro grupo del paralelo anterior que no utilizó esta herramienta para esta unidad. El análisis estadístico se basó en el parámetro chi-cuadrado, partiendo de la hipótesis nula que las calificaciones de estos dos grupos estudiados eran por azar ya que no dependen del uso de la aplicación Socrative. Para ello, se consideró el valor de $p$, pues si $p<.05$ se rechazaba la hipótesis nula y se concluía que las calificaciones estaban estrechamente relacionadas con el uso del Socrative, lo que indicaba la existencia de diferencias significativas en las notas de ambos grupos.

\subsubsection{En cuanto al grado de satisfacción}

Para medir la experiencia del uso del Socrative como herramienta de evaluación y aprendizaje, se consideró la opinión del estudiantado mediante una encuesta de satisfacción. Se aplicó el coeficiente de alfa de Cronbach para evaluar la confiabilidad de dicho instrumento y estadística descriptiva para visualizar la frecuencia y porcentaje del aporte del uso del Socrative en cada uno de los criterios. De esta manera, cada estudiante valoró a la encuesta entre 20 y 100 puntos mientras que el grado de satisfacción del uso del Socrative en cada criterio lo valoró entre 36 y 180 puntos, ubicándolo en el siguiente baremo: Inaceptable (36 a 77 puntos), Moderadamente Aceptable (78 a 118 puntos), Aceptable (119 a 159 puntos), Sobresaliente (160 a 180 puntos).

\subsubsection{En cuanto a la variable uso del Socrative como herramienta para la evaluación y aprendizaje}

Para cuantificar a la variable de la investigación se consideraron los resultados de cada uno de los tres cuestionarios junto con la encuesta, los cuales están relacionados con los niveles de conocimientos y el grado de satisfacción. Para ello, se sumaron las puntuaciones de los cuestionarios y de la encuesta por estudiante, obteniendo puntuaciones finales que oscilaban entre 20 y 130 puntos, para luego ubicar los resultados sobre el uso del Socrative como herramienta para la evaluación y el aprendizaje en el siguiente baremo: Deficiente (20 a 48 puntos), Debe mejorar (49 a 75 puntos), Satisfactorio (76 a 103 puntos), Excelente (104 a 130 puntos). 


\section{Resultados y su Discusión}

\subsection{Resultados para el nivel de conocimientos previos}

Para medir el nivel de conocimientos previos se consideraron los resultados del cuestionario inicial (test). Dichos resultados se clasificaron en los 5 niveles de aprendizaje de acuerdo con los modelos de pensamiento del estudiantado. En la tabla 1 se puede observar que el $69,4 \%$ del estudiantado se ubicó en el nivel de aprendizaje B por lo tanto tiene un alto nivel de conocimientos para el inicio de la unidad Funciones. De igual manera, es notable en dicha tabla que el nivel de conocimiento del 97,2\% del estudiantado (35 estudiantes) está agrupado en los tres más altos niveles de aprendizaje. En consecuencia, se demuestra que la clase tiene un nivel de conocimientos previos satisfactorio para la unidad funciones.

Tabla 1

Resultados expresados en forma de frecuencia y porcentaje agrupados en los niveles de aprendizajes correspondientes a los cuestionarios aplicados

\begin{tabular}{lrrrrrr}
\hline \multirow{2}{*}{ Cuestionarios } & \multicolumn{7}{c}{ Niveles de Aprendizaje } \\
\cline { 2 - 7 } & A & B & C & D & E & Total \\
\hline Cuestionario Inicial & & & & & & \\
\hline Frecuencia & 5 & 25 & 5 & 0 & 1 & 36 \\
Porcentaje & 13,9 & 69,4 & 13,9 & 0,0 & 2,8 & 100 \\
\hline Cuestionario Intermedio & & & & & & \\
\hline Frecuencia & 2 & 12 & 13 & 8 & 1 & 36 \\
Porcentaje & 5,6 & 33,3 & 36,1 & 22,2 & 2,8 & 100 \\
\hline Cuestionario Final & & & & & & \\
\hline Frecuencia & 27 & 4 & 2 & 0 & 3 & 36 \\
Porcentaje & 75,0 & 11,1 & 5,6 & 0,0 & 8,3 & 100
\end{tabular}

Fuente: Elaboración propia, 2021

Estos hallazgos difieren de los resultados de Santos et al. (2016), cuyos alumnos mostraron escasos saberes al inicio de la clase, ubicándose en los niveles más bajos de aprendizaje. No obstante, coincide con lo expuesto por Pérez (2019) quien indica que a medida que el nivel de conocimiento previo es mayor, al alumnado se le hará más fácil tener contacto con un nuevo saber; es decir mayor posibilidad de construir un nuevo significado, pues contará con los fundamentos apropiados para la creación de los mismos, ya que esta actividad mental constructiva no puede partir del vacío, de la nada. Asimismo, la retroalimentación de los resultados que ofrece esta plataforma, le permite al profesorado diagnosticar y aprovechar para aclarar dudas y corregir errores cognitivos sobre la introducción del tema funciones. 


\subsection{Resultados para el nivel de avance}

Para medir el nivel de avance del estudiantado se consideraron los resultados del cuestionario intermedio (preguntas de respuestas cortas). Dichos resultados se clasificaron en los 5 niveles de aprendizaje de acuerdo con los modelos de pensamiento del estudiantado. En la tabla 1 se puede visualizar que el 36,1\% del estudiantado se ubicó en el nivel de aprendizaje C por lo que presentan un nivel medio de avance en el aprendizaje de la unidad Funciones. Asimismo, en dicha tabla se puede concluir que el $75 \%$ del estudiantado está distribuido entre los tres niveles más alto de aprendizaje ( $A, B$ y $C)$.

Ahora bien, mediante los resultados de la prueba McNemar se realizaron los cruces del nivel de conocimientos previos y del nivel de avance en estudiantes. De la tabla 2 se observa que 23 estudiantes $(63,9 \%)$ presentaron un nivel de avance del aprendizaje satisfactorio ante 13 estudiantes $(36,1 \%)$ cuyo nivel resultó insatisfactorio. Sin embargo, en la misma tabla se distingue que 12 estudiantes (34,3\%) que originalmente presentaron un nivel de conocimientos previos satisfactorio, luego de realizarse la intervención (uso del Socrative), esta proporción del alumnado presentó un nivel insatisfactorio de avance. En este sentido, para este grupo estudiantil no se puede considerar como un éxito del tratamiento que buscaba mejorar el nivel de conocimiento del estudiantado. Aunado a ello, la proporción del estudiantado (un estudiante) que presentó un nivel de conocimiento previo insatisfactorio no mejoró luego de la intervención pedagógica, ya que siguió manteniendo un nivel de avance insatisfactorio.

Tabla 2

Tabulación cruzada entre Nivel de conocimiento previo y Nivel de avance de aprendizaje del estudiantado

\begin{tabular}{lrr} 
Nivel de conocimiento & \multicolumn{3}{c}{ Nivel de avance de aprendizaje } \\
\cline { 2 - 3 } previo & Insatisfactorio & Satisfactorio \\
\hline Insatisfactorio & 1 & 0 \\
\hline Satisfactorio & 12 & 23 \\
\hline
\end{tabular}

Fuente: Elaboración propia, 2021

Es importante destacar que este tipo de preguntas (preguntas de respuestas cortas) fueron las menos contestadas por el alumnado. Estos resultados concuerdan con los hallazgos de Rodríguez et al. (2017), quienes manifestaron que este tipo de preguntas suelen ser las menos acertadas pues si el alumnado no conoce la respuesta con cierto nivel de confianza prefiere no arriesgarse; además el estudiantado no tiene la opción de seleccionar una respuesta por lo tanto no cuenta con el azar. 
Una vez conocido los resultados anteriores, mediante la estadística de la prueba de McNemar, se podrá determinar si la proporción del estudiantado que mostró cambios en su nivel de conocimiento, luego del tratamiento, presenta una diferencia estadísticamente significativa. Para este caso, se consideró como hipótesis nula que, el nivel de conocimientos previos en estudiantes universitarios no induce cambios en el nivel de avance de aprendizaje en el estudiantado universitario que utiliza Socrative. Mientras que la hipótesis alternativa era que, el nivel de conocimientos previos en estudiantes universitarios si induce cambios en el nivel de avance del aprendizaje en el estudiantado universitario que utiliza Socrative. Al aplicar el estadístico de contraste en la prueba de McNemar se obtuvo que $p<.001$ (ver tabla 3), entonces se determinó que el cambio en el nivel de avance del estudiantado después de la intervención pedagógica (uso del Socrative) fue estadísticamente significativo.

Tabla 3

Estadísticos de contraste $e^{a}$ de la Prueba de McNemar para Nivel de conocimiento previos y. Nivel de avance en el aprendizaje del estudiantado Nivel de conocimiento previo y Nivel de avance de aprendizaje

\begin{tabular}{lr}
\hline $\mathrm{N}$ & 36 \\
Sig. exacta (bilateral) & $.00049^{\mathrm{b}}$ \\
\hline
\end{tabular}

a. Prueba de McNemar

b. Se ha usado la distribución binomial.

Fuente: Elaboración propia, 2021

En líneas generales, estos resultados coinciden con Santos et al. (2016) ya que a medida que transcurrían las clases aumentaba el nivel de aprendizaje a pesar de la dificultad del tipo de pregunta para el estudiantado. En este sentido, según Asadovay y Morocho (2015), cuando un alumno presenta un buen dominio de los conocimientos anteriormente adquiridos facilita su aprendizaje en la medida en que estos saberes representan puentes cognitivos para alcanzar aprendizajes significativos basándose en una buena articulación o integración entre estos conocimientos previos y la nueva información.

Para Bizarro et al. (2019), durante el desarrollo de la unidad fue el momento para que el aprendiz, por medio de la retroalimentación, mejorará su aprendizaje enfocándose en sus fortalezas y debilidades. Mientras que para el profesorado representó el momento de repasar o reforzar los contenidos necesarios, así como ofrecerle al alumnado los medios para superar sus dificultades. 


\subsection{Resultados para el nivel de comprensión}

Para medir el nivel de comprensión general del estudiantado se consideraron los resultados del cuestionario final (carrera espacial). Tales resultados se clasificaron en los 5 niveles de aprendizaje de acuerdo con los modelos de pensamiento del estudiantado. En la tabla 1 se puede observar que el $75 \%$ del estudiantado se ubicó en el nivel de aprendizaje A por lo que presenta un muy alto nivel de comprensión en el aprendizaje de la unidad Funciones.

La prueba de McNemar permitió realizar el cruce entre el nivel de avance de aprendizaje y el nivel de comprensión general del estudiantado. La tabla 4 muestra que el nivel de comprensión de 31 estudiantes $(86,1 \%)$ fue satisfactorio mientras que para 5 estudiantes $(13,9 \%)$ resultó insatisfactorio.

Sin embargo, en la misma tabla se destaca que 10 estudiantes $(34,3 \%)$ que originalmente presentaron un nivel de avance insatisfactorio, presentaron un nivel de comprensión satisfactorio luego de realizarse la intervención (uso del Socrative). En este sentido, este grupo estudiantil puede considerarse como un éxito del tratamiento que buscaba mejorar el nivel de conocimiento en general del estudiantado sobre la unidad funciones. No obstante, dos estudiantes que presentaron un nivel de avance satisfactorio, después de la intervención, obtuvieron un nivel de comprensión insatisfactorio. En general, se presentó una mayor proporción de cambios positivos que negativos en el nivel de conocimiento del estudiantado, una vez que fueron sometidos a la intervención pedagógica.

Tabla 4

Tabulación cruzada entre Nivel de avance de aprendizaje y Nivel de comprensión del estudiantado

\begin{tabular}{lrrr}
\hline \multirow{2}{*}{ Nivel de avance de } & \multicolumn{3}{c}{ Nivel de comprensión } \\
\cline { 2 - 4 } aprendizaje & Insatisfactorio & Satisfactorio \\
\hline Insatisfactorio & 3 & 10 \\
\hline Satisfactorio & 2 & 21 \\
\hline Fuente: Elaboración propia, 2021 & &
\end{tabular}

Estos resultados coincidieron con los hallazgos de Narbón y Peiró (2018) y Parra et al. (2017) quienes mostraron que es precisamente al final o cierre de la unidad, el momento de la clase cuando el alumnado tiene un mayor control del contenido de los temas pues ya ha recibido una retroalimentación sobre errores cometidos y ha superado sus debilidades y fallas teóricas. Del mismo modo, estos mismos autores también señalaron que debido a que la última actividad fue de competencia en el que se generó un ambiente con una alta motivación y 
participación activa, el estudiantado trato de no equivocarse leyendo atentamente cada pregunta para luego reflexionar sobre ella. Por tanto, cada estudiante se esforzó para poder pertenecer al grupo de vencedores.

Asimismo, mediante los resultados de la prueba McNemar, se realizaron los cruces del nivel de avance del estudiantado y el nivel de comprensión. Para esta prueba la hipótesis nula fue que, el nivel de avance en el aprendizaje estudiantil universitario no induce cambios en el nivel de comprensión del estudiantado universitario al utilizar Socrative. Mientras que la hipótesis alternativa era que, el nivel de avance en el aprendizaje del estudiantado universitario si induce cambios en el nivel de comprensión estudiantil universitario con el uso del Socrative. El estadístico de contraste en la prueba de McNemar indicó que: $p=.039$ (ver tabla 5), por lo tanto el cambio en el nivel de comprensión del estudiantado después de la intervención pedagógica (uso del Socrative) fue estadísticamente significativo.

Tabla 5

Estadísticos de contraste ${ }^{a}$ de la Prueba de McNemar para Nivel de avance en el aprendizaje y Nivel de comprensión Nivel de avance y Nivel de comprensión $\mathrm{N}$

Sig. exacta (bilateral) , $039 \mathrm{~b}$

a. Prueba de McNemar

b. Se ha usado la distribución binomial. Fuente: Elaboración Propia, 2021

Los resultados coinciden con Grueso et al. (2016), quienes indicaron que al monitorizar el aprendizaje del estudiantado con la finalidad de ofrecerle una retroalimentación permanente le permite al alumnado mejorar su aprendizaje y obtener altos rendimientos académicos; asimismo el profesorado tendrá la oportunidad de modificar o perfeccionar sus estrategias. Bajo estas circunstancias, al término del proceso de aprendizaje de la unidad funciones se verificó que el alumnado cumplió con los comportamientos terminales trazados por el grupo profesoral y que cuenta con los conocimientos necesarios para posteriores aprendizajes.

\subsection{Resultados en cuanto a la metodología empleada en las clases}

Al evaluar si el uso del Socrative como herramienta de evaluación y aprendizaje, se asocia con el resultado del examen final de funciones (aprobado o aplazado), se consideraron las calificaciones de dos grupos y se aplicó el parámetro chi-cuadrado, partiendo de la hipótesis nula que no existe relación entre el resultado del examen final del estudiantado y el 
uso del Socrative (son independientes) y como hipótesis alternativa que existe relación entre el resultado del examen final del estudiantado y el uso del Socrative (son dependientes). Los resultados de la prueba de chi-cuadrado fueron: $\chi^{2}(1, N=36)=0.13, p=.72$; por lo tanto existe suficiente evidencia para afirmar que el resultado del examen final de la unidad funciones y el uso del Socrative no están relacionados (se rechaza la dependencia entre ellas).

Los resultados sobre el porcentaje de aprobación indicaron que el grupo que utilizó Socrative obtuvo un porcentaje mayor, el 91,87\%, aunque no resultó tan relevante en comparación con el porcentaje del grupo que no utilizó Socrative que fue del 89,74\%; ocurriendo la misma situación con los promedios de ambos cursos ya que el promedio del grupo con Socrative fue de 15,13 puntos mientras que el que no lo uso fue de 13,79 puntos. Por lo tanto, de manera global la diferencia en las calificaciones del alumnado que han utilizado o no han utilizado esta plataforma no es significativa.

Estos hallazgos coinciden con los presentados por Arraiga et al. (2017), Cosi y Voltas (2019) y Santos et al. (2016), quienes concluyeron que, con el uso del Socrative, el estudiantado incrementó su nivel de motivación y participación, así como su proceso de aprendizaje aunque éste con menor proporción, ya que la diferencia no fue significativa entre el grupo tradicional y los que utilizaron Socrative pero lograron mejorar las competencias programadas en la unidad.

\subsection{Resultados sobre el grado de satisfacción del uso del Socrative}

Los resultados que se muestran en esta sección resumen la opinión del alumnado sobre la experiencia de utilizar Socrative como herramienta de evaluación y aprendizaje, se tomó en cuenta el aporte de esta plataforma en cada uno de los criterios pedagógicos considerados en esta investigación. En la figura 2 se exponen los resultados relacionados con la ubicación en el baremo de cada criterio. El $90 \%$ de los criterios se ubican en la categoría de Aceptable, mientras que sólo el 10\% (2 criterios) se ubicaron en la categoría de Sobresaliente, tales criterios fueron el desarrollo de habilidades y el trabajo autónomo con una puntuación de 160 puntos cada uno. 
Figura 2

Criterios pedagógicos del Uso del Socrative según el estudiantado universitario de la Universidad Particular de Loja en Ecuador, periodo 2018-2019.

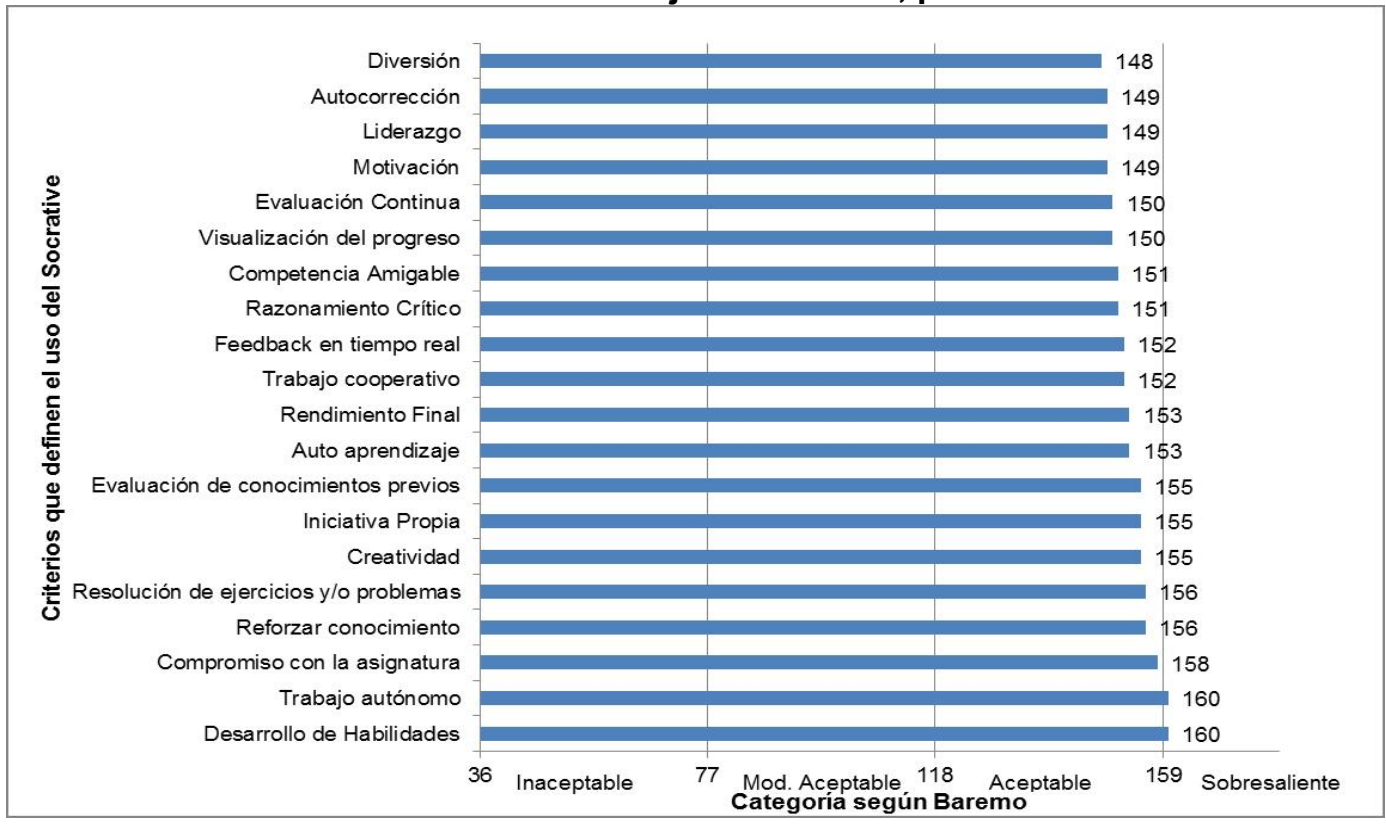

Fuente: Elaboración Propia, 2021

Estos resultados concuerdan con lo planteado por Cosi y Voltas (2019) al afirmar que con el uso de este tipo de metodologías se enriquece el aprendizaje favoreciendo el desarrollo de competencias y habilidades. Asimismo, se cumplió lo indicado por Perera y Hervás (2019) y por Moya y Soler (2018) en lo referente a la capacidad que tiene esta herramienta para crear escenarios de formación en el que el grupo estudiantil sea el protagonista de su aprendizaje, siendo el trabajo autónomo clave para que este grupo pueda supervisar y autorregular su propio aprendizaje, para favorecer el trabajo personal y lograr una experiencia más personalizada.

De conformidad con los resultados obtenidos, Rodríguez et al. (2017) y López et al. (2019) consideraron que estas plataformas incentivan principalmente la motivación lo cual genera interés y compromiso con la asignatura, por lo que el estudiantado debe llevar la materia al día promoviendo una participación más activa. Sin embargo, para Masero (2019) y Puche (2018) la razón principal del uso de esta estrategia digital es reforzar, repasar, asimilar y discutir los diferentes contenidos analizados en las clases para detectar errores cognitivos en tiempo real y así impulsar algunos cambios de estrategias por parte del profesorado; aunque para este estudio este criterio no resultó ser el más sobresaliente. 


\subsection{Resultados para la variable Uso del Socrative como herramienta para la evaluación y aprendizaje}

Los resultados relacionados con la variable de investigación, Uso del Socrative como herramienta para la evaluación y el aprendizaje; indicaron que un $66,7 \%$ del estudiantado la consideraron sobresaliente, un $30,5 \%$ como satisfactorio y un $2,8 \%$ expresaron que debe mejorar su uso como herramienta de apoyo en el proceso de evaluación y aprendizaje.

Entre las sugerencias del estudiantado para mejorar y optimizar esta herramienta pedagógica destacaron: crear una sección para sugerir ítems para el diseño de las preguntas de los cuestionarios, anexar niveles de dificultad según el progreso del alumnado, agregar pistas que ayuden al estudiantado de acuerdo al grado de dificultad del ítem, incluir recompensas para reforzar la motivación en la actividad.

Los resultados asociados con la variable de estudio, se ajustaron plenamente con los estudios de Basso y Loyer (2016) y Calderón et al. (2019), en los que el uso del Socrative favoreció tanto al profesorado, desde el punto de vista metodológico y evaluativo, como al estudiantado en su aprendizaje contribuyendo a mejorar el rendimiento académico y a la renovación pedagógica, pues estas herramientas deben utilizarse dentro de la labor docente como un medio no como un fin.

Asimismo, se evidenció lo expresado por Ruíz (2019) al afirmar que este tipo de actividades brindan un extenso abanico de posibilidades en la que la competición sana es parte de su bandera para captar la atención y lograr motivar al grupo estudiantil a ser más responsable de su formación. De esta manera, para González-Fernández y Salcines-Talledo (2015) y Frías et al. (2016) la aplicación de estas plataformas digitales mediante la integración de móviles al aula, resultaron fundamentales para fortalecer el aprendizaje por medio de una retroalimentación continua y en tiempo real. En este mismo orden de ideas, Puche y Pérez (2018) opinaron que las actividades basadas en el juego representan una estrategia didáctica beneficiosa para el área de las matemáticas pues logran un ambiente de clase más dinámico, divertido y participativo lo cual permite incrementar una relación positiva entre estudiantedocente.

\section{Conclusiones}

Según los resultados anteriormente expuestos, se puede concluir que el éxito de Socrative como herramienta didáctica radica en que permite tanto al estudiantado como al profesorado reforzar contenidos, enmendar errores cognitivos, realizar autoevaluaciones y 
autocorrecciones. Además, con la incorporación de elementos lúdicos en la clase se logra incrementar la participación del estudiantado. Asimismo, el profesorado con este tipo de recurso puede diagnosticar saberes previos del estudiantado, el grado de avance durante el desarrollo de la clase así como el nivel de asimilación de las competencias programadas durante el aprendizaje, lo que le permitirá al profesor realizar cambios en sus estrategias de enseñanzas de ser necesario.

Definitivamente, la evaluación en tiempo real contribuye enormemente con el proceso de enseñanza debido a sus bondades al aplicarse en diversos momentos cruciales (inicio, desarrollo y cierre) del desarrollo de la unidad Funciones. De esta manera, el cuerpo docente podrá asumir un papel preventivo para asegurar un aprendizaje significativo y el estudiantado podrá admitir su responsabilidad ante sus debilidades y fortalezas para garantizar su aprendizaje. Según los resultados, el estudiantado de la asignatura Fundamentos matemáticos presentaron un nivel de conocimientos previos satisfactorio lo cual ayudó a que tanto el nivel de avance como el nivel de comprensión del contenido de la unidad funciones fueran buenos e incluso significativos. Las calificaciones, como dato cuantitativo, indicaron que el rendimiento académico del curso fue bueno.

Bajo estas circunstancias, se puede deducir que son diversos los beneficios que aporta el uso del Socrative en cada uno de los criterios en los que interviene. El grupo estudiantil manifestó que esta herramienta didáctica resultó ser eficiente y sobresaliente para el proceso de aprendizaje de contenidos relacionados con la unidad funciones. De hecho, para este estudiantado el empleo de esta estrategia virtual representó una experiencia educativa más activa y participativa, en la que el desarrollo de habilidades, así como el trabajo autónomo fueron los criterios más destacados.

De esta manera, con la implementación de herramientas didácticas como Socrative en las aulas universitarias, se evidencia la eficiencia en el proceso de formación tanto para el estudiantado como para el profesorado en el campo de las matemáticas universitarias. Sin duda alguna, el perfil de un estudiantado inmerso dentro de una era digital facilita la decisión del profesorado de incorporar este tipo de recursos dentro del aula mediante dispositivos móviles. Ahora bien, otra de las ventajas para implementar este tipo de innovaciones en clases, es que en la Universidad Técnica Particular de Loja se puede acceder a internet desde las aulas y además Socrative es una plataforma de acceso gratuito. Aunado a lo anterior, casi el $100 \%$ del alumnado de la asignatura poseía un dispositivo móvil para realizar este tipo de actividades, por lo que no representó una limitante para la experiencia ni tampoco para 
descartar su implementación en la enseñanza de otras unidades de la misma materia o, inclusive, en otras asignaturas.

Obviamente, es parte de la responsabilidad docente involucrarse en este tipo de innovaciones pedagógicas lo cual dependerá del objetivo educativo que se pretenda alcanzar, lo que le permitirá la selección de la herramienta didáctica adecuada de una variedad de opciones que existen en la actualidad. Por esta razón, se sugiere alternar estas plataformas para evitar el aburrimiento y perder el nivel de atención que se logra en el alumnado cuando comienza a utilizar este tipo de estrategias.

Finalmente, se puede asegurar que estas propuestas didácticas cuentan con un gran potencial pedagógico, ya que los dispositivos móviles representan recursos propicios en la mejora del proceso de enseñanza aprendizaje de contenidos matemáticos pues facilitan el diseño de actividades que promuevan un mayor desempeño estudiantil y permitan el monitoreo de la enseñanza, aprendizaje y evaluación cubriendo las necesidades educativas del cuerpo estudiantil.

Hay que considerar que, a pesar que en esta investigación no se presentaron inconvenientes desde el punto de vista de infraestructura ni plataforma tecnológica, hubo una limitante al momento de recolectar la información de la encuesta dirigida al estudiantado, ya que, aunque ellos manifestaron su disposición de llenarla, se llevó más del tiempo estipulado para esa etapa. Asimismo, sólo participó uno de los dos docentes asignados en esa asignatura durante ese ciclo lectivo del semestre.

Concretamente, con esta investigación se busca, en general, ofrecer información valiosa a estudiantes y docentes sobre la aplicación de nuevas prácticas educativas, caracterizadas por ser divertidas, eficientes y capaces de adaptarse a las exigencias del aula universitaria siendo el estudiantado el centro de su propio proceso de formación. De este modo, se pretende abonar el camino a nuevas investigaciones con diferentes herramientas de este tipo para luego comparar resultados que nos indiquen el grado de satisfacción de los actores involucrados y el nivel de eficacia de cada una de estas estrategias en el proceso de enseñanza, aprendizaje y evaluación de contenidos matemáticos universitarios. 


\section{Referencias}

Arraiga, María., González, María., y López, Luis. (2017). Evaluación del aprendizaje significativo con la aplicación Socrative. Tecnología Educativa Revista Conaic, 4(2), 5257. https://conaic.net/revista/publicaciones/Vol IV Num2 2017/Articulo 4.pdf

Arribas, José. (2017). La Evaluación de los Aprendizajes. Problemas y Soluciones. Profesorado. Revista de Currículum y Formación de Profesorado, 21(4), 381-404. http://www.redalyc.org/articulo.oa?id=56754639020

Asadovay, Diego., y Morocho, Luis. (2015). La activación de los conocimientos previos para lograr un aprendizaje significativo en niños de E.G.B. (Tesis de pregrado). Universidad de Cuenca, Cuenca, Ecuador. https://dspace.ucuenca.edu.ec/bitstream/123456789/23183/1/Tesis.pdf

Basso, Matilde., y Loyer, Solange. (2016). Uso de Socrative como elemento de interacción en cursos de Ingeniería. XXIX Congreso Chileno de Educación en Ingeniería UFRO 2016, Chile. content/uploads/2016/10/SOCHEDI 2016 paper 26 VF.pdf

Bizarro, Wilfredo., Sucari, Wilson., y Quispe-Coaquira, Angela. (2019). Evaluación formativa en el marco del enfoque por competencias. Revista Innova Educación, 1(3), 374-390. DOI: https://doi.org/10.35622/j.rie.2019.03.r001

Calderón, Susana., García, Francisco., y Rodríguez, Beatriz. (14-15 de noviembre de 2019). Eficiencia de la metodología de la clase invertida en la enseñanza universitaria. VI Jornadas Iberoamericanas de Innovación Educativa en el Ámbito de las TIC y las TAC, Las Palmas de Gran Canaria, España.

Cosi, Sandra., y Voltas, Núria. (2019). Evaluación formativa en estudiantes universitarios mediante tecnologías digitales: el rol del alumno en su propio proceso de enseñanzaaprendizaje. En Rosabel Roig-Vila (Ed.), Investigación e innovación en la Enseñanza Superior. Nuevos contextos, nuevas ideas (pp. 113-123). Octaedro. https://rua.ua.es/dspace/bitstream/10045/98852/1/Investigacion-e-innovacion-en-laES 011.pdf

Chamorro, Cristina., Bejarano, Silvia., y Colcha, Esthela. (2021). SOCRATIVE, aplicación interactiva para evaluar los resultados de aprendizaje en los estudiantes de inglés como Lengua Extranjera. Dominio de las Ciencias, 7(1), 849-873. https://dominiodelasciencias.com/ojs/index.php/es/article/view/1681/3281

Díaz, Camilo., Rosero, Karol., y Obando, Myriam. (2018). La evaluación como medio de aprendizaje. Educación y Humanismo, 20(34), 173-186. http://dx.doi.org/10.17081/eduhum.20.34.2863

Fernández, Sonsoles. (2017). Evaluación y Aprendizaje. Marco ELE Revista de didáctica español como lengua extranjera, https://marcoele.com/descargas/24/fernandez-evaluacion aprendizaje.pdf 
Fies, Carmen., and Marshall, Jill. (2006). Classroom Response Systems: A Review of the Literature. Journal Of Science Education And Technology, 15(1), 101-109. DOI: https://doi.org/10.1007/s10956-006-0360-1

Frías, María., Arce, Christian., y Flores, Patricio. (2016). Uso de la plataforma socrative.com para alumnos de Química General. Educación química, 27(1), 59-66. DOI: https://dx.doi.org/10.1016/j.eq.2015.09.003

González-Fernández, Natalia., y Salcines-Talledo, Irina. (2015). El Smatphone en los procesos de enseñanza aprendizaje-evaluación en Educación Superior. Percepciones de $\begin{array}{lllll}\text { docentes y } & \text { estudiantes. RELIEVE, 21(2), } & \text { 1-20. }\end{array}$ http://dx.doi.org./10.7203/relieve.21.2.7480

Grueso, Elia., Pérez, Pilar., y Prado, Rafael. (2016). Aprendizaje significativo del alumnado de física aplicada del grado en farmacia: evaluación basada en el empleo de cuestionarios. Ars Pharmaceutica, 55(4), 8-13. https://bit.ly/3lxzXkt

Jaber, J. Raduan., Farray, David., Melian, Carlos., Ramírez, Ana., Suárez, Francisco., Rodríguez, Eligia., Suarez-Bonnet, Alejandro., y Carrascosa, Conrado. (15 - 16 de noviembre de 2018). Comparación de dos herramientas de gamificación para el aprendizaje en la docencia universitaria. V Jornadas Iberoamericanas de Innovación Educativa en el Ámbito de las TIC y las TAC, Las Palmas de Gran Canaria, España.

Jordán, Angela., Morán, Lila., y Camacho, Gina. (2018). La evaluación de los aprendizajes y su influencia en la calidad del proceso de enseñanza aprendizaje en el contexto $\begin{array}{llll}\text { universitario. } & \text { Opuntia } & \text { Brava, } & \text { 215-224. }\end{array}$ http://opuntiabrava.ult.edu.cu/index.php/opuntiabrava/article/view/134

Joya, María. (2020). La evaluación formativa, una práctica eficaz en el desempeño docente. Revista Scientific, 5(16), 179-193. DOI: https://doi.org/10.29394/Scientific.issn.25422987.2020.5.16.9.179-193

López, Carmen., Azaustre, María., y Rodríguez, Emma. (2019). Instrumentos de Innovación Docente en la Enseñanza-Aprendizaje del Derecho Romano. Revista Internacional de Derecho Romano, (23),

454-491. https://dialnet.unirioja.es/servlet/articulo?codigo $=7149426$

López, Fernando y Silva, María. (2016). Factores que inciden en la aceptación de los dispositivos móviles para el aprendizaje en educación superior. Estudios sobre educación, 30, 175-195. DOI: https://doi.org/10.15581/004.30.175-195

López, José., Varo, Marta., y Pontes, Alfonso. (2017). Uso de sistemas de respuesta inmediata para mejorar el aprendizaje de conceptos de termodinámica en la Universidad (5-8 de septiembre). X Congreso Internacional sobre Investigación en Didáctica de las Ciencias, Sevilla, España. https://raco.cat/index.php/Ensenanza/article/view/335523/426355

Masero, Inmaculada. (2019). Móviles y aprendizaje en la educación superior. 3C TIC. Cuadernos de desarrollo aplicados a las TIC, 8(4), 13-25. DOI: https://doi.org/10.17993/3ctic.2019.84.13-25 
Mateus, Julio., Aran, Sue., y Masanet, María. (2017). Análisis de la Literatura sobre Dispositivos Móviles en la Universidad Española. RIED. Revista Iberoamericana de Educación a Distancia, 20(2), 49-72. DOI: http://dx.doi.org/10.5944/ried.20.2.17710

Moya, María., y Soler, Carolina. (2018). La gamificación mediante herramientas virtuales de respuesta de audiencia; La experiencia de socrative y kahoot. En Rosabel Roig-Vila (Ed.), El compromiso académico y social a través de la investigación e innovación educativas en la Enseñanza Superior (1154-1163). Octaedro. http://rua.ua.es/dspace/handle/10045/88088

Narbón, Isabel., y Peiró Jesús. (2018). La plataforma Socrative como herramienta de aprendizaje: Una aplicación a la asignatura Métodos Cuantitativos. Revista electrónica sobre la enseñanza de la Economía Pública, (22), 41-50. http://epublica.unizar.es/es/articulo/la-plataforma-socrative-herramienta-aprendizaje-unaaplicacion-la-asignatura-metodos-cuantitativos

Parra, Teresa., Molina, José., Luna, Gabriel., Milanovic, Ivana., Casanova, Gerard., y Castro, Francisco. (2017). La aplicación SOCRATIVE como herramienta de evaluación y precursor de la participación en el aula. En Rosabel Roig-Vila (Ed.), Investigación en docencia universitaria. Diseñando el futuro a partir de la innovación educativa (pp. 667683). Octaedro. https://rua.ua.es/dspace/bitstream/10045/71188/1/Investigacion-endocencia-universitaria 70.pdf

Perera, Victor., y Hervás, Carlos. (2019). Percepción de estudiantes universitarios sobre el uso de Socrative en experiencias de aprendizaje con tecnología móvil. Revista Electrónica de Investigación Educativa, 21(e05), 1-10. DOI: https://doi.org/10.24320/redie.2019.21.e05.1850

Pérez, Alejandro. (2019). Conocimientos previos e intervención docente. Revista Acta Educativa, 1-30. https://revista.universidadabierta.edu.mx/docs/Conocimientos $\% 20$ previos $\% 20 \mathrm{e} \% 20$ inte rvenci\%C3\%B3n\%20docente.pdf

Pianucci, Irma., y Tenutto, Marta. (2020). Potencial de la tecnología digital para la retroalimentación efectiva en diferentes momentos evaluativos. Congreso Iberoamericano La educación ante el nuevo entorno digital. Madrid, España.

Puche, Javier. (2018). Métodos y aplicaciones innovadoras para potenciar la evaluación del aprendizaje en la docencia universitaria. En REDINE (Ed.), Innovative strategies for Higher Education in Spain (131-140). Adaya Press. http://www.adayapress.com/wpcontent/uploads/2018/04/ched14.pdf

Puche, Javier., y Pérez, Pedro. (7-8 de junio de 2018). Estrategias y aplicaciones innovadoras para potenciar el proceso de aprendizaje de la Historia Económica. XIII Encuentro de Didáctica de la Historia Económica. Universitat de les Illes Balears, Palma de Mallorca, España.

https://congresodidactica2018.uib.es/digitalAssets/493/493948 cxiii didactica comunic aciones-compressed.pdf 
Rapún, Marta., y Rapún, Carlos. (2018). Evaluación Cooperativa en Educación Física mediante el Socrative.com. EmásF, Revista Digital de Educación Física, (50), 56-61. https://dialnet.unirioja.es/servlet/articulo?codigo $=6266672$

Rodríguez, María., Barrallo, Alejandro., Franch, Angels., Castell, Margarida., y Pérez, Francisco. (2017). Amenizar las clases de Fisiología con la aplicación Socrative. Fundación Educación Médica, 20(1), 64-69. https://scielo.isciii.es/pdf/fem/v20s1/20149832-fem-20-s1-s64.pdf

Rodríguez, Liliana., y Gracia, Edison. (2016). Estrategias y criterios de evaluación que aplican los profesores en el proceso de enseñanza-aprendizaje. Dominio de las Ciencias, 2(Extra), 59-74. https://dialnet.unirioja.es/servlet/articulo?codigo $=5761546$

Ruíz, Salvador. (2019). Gamificación como estrategia de evaluación de Química en alumnos de bachillerato. XV Congreso Nacional de Investigación Educativa COME 2019. México. http://www.comie.org.mx/congreso/memoriaelectronica/v15/doc/0004.pdf

Saltos-Dueñas, Celia., y Chiriboga-Zambrano, Marjorie. (2016). La evaluación formativa en el desempeño de los estudiantes. Dominio de las Ciencias, 2(Esp), 112-127. https://dominiodelasciencias.com/ojs/index.php/es/article/viewFile/88/79

Sánchez, Melchor. (2018). La evaluación del aprendizaje de los estudiantes: ¿es realmente tan complicada?. Revista Digital Universitaria, 19(6), 1-18. DOI: http://doi.org/10.22201/codeic.16076079e.2018.v19n6.a1

Santos, Jennifer., Grueso, Elia., y Trujillo, Luis. (2016). Uso de una aplicación móvil para aumentar la motivación del alumnado en formulación y nomenclatura química. AFINIDAD LXXIV, 576, 278-284. https://raco.cat/index.php/afinidad/article/view/318420/408590

Subirats, Xavier. (2018). Socrative, una herramienta interactiva para el aprendizaje y la evaluación a través del móvil. Actualidad Analítica, 63, 7-10. https://www.seqa.es/ActualidadAnalitica/AA 63/0002 Xubirats.pdf

Zamora, Ronald. (2019). El m-learning, las ventajas de la utilización de dispositivos móviles en el proceso autónomo de aprendizaje. ReHuSo: Revista de Ciencias Humanísticas y Sociales, 4(3), 29-38. https://dialnet.unirioja.es/servlet/articulo?codigo=7047179 
Revista indizada en

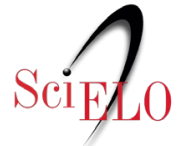

redalyc.sy latindex

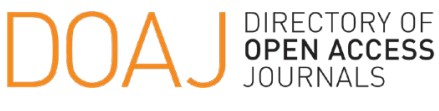

Distribuida en las bases de datos:
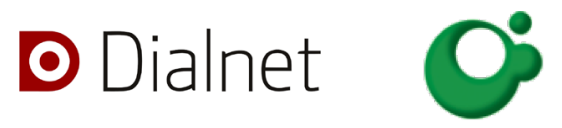
SHERPA/RøMEO

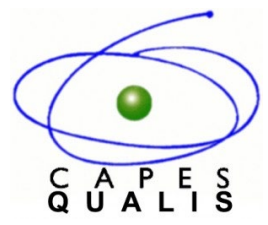

MIAR 